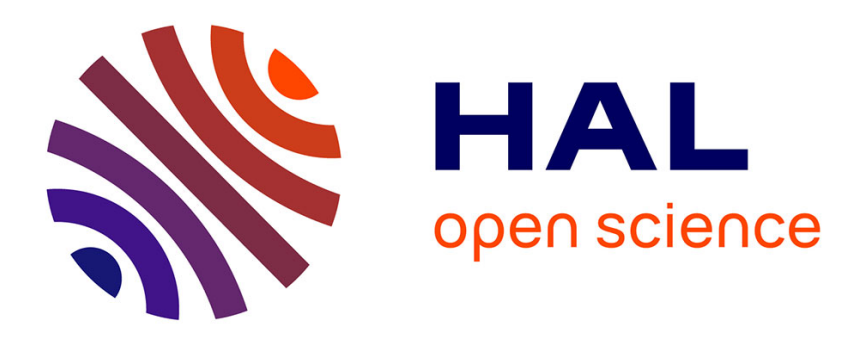

\title{
A Lemke-like algorithm for the Multiclass Network Equilibrium Problem
}

Frédéric Meunier, Thomas Pradeau

\section{To cite this version:}

Frédéric Meunier, Thomas Pradeau. A Lemke-like algorithm for the Multiclass Network Equilibrium Problem. 2013. hal-00857611

\section{HAL Id: hal-00857611 \\ https://hal.science/hal-00857611}

Preprint submitted on 3 Sep 2013

HAL is a multi-disciplinary open access archive for the deposit and dissemination of scientific research documents, whether they are published or not. The documents may come from teaching and research institutions in France or abroad, or from public or private research centers.
L'archive ouverte pluridisciplinaire HAL, est destinée au dépôt et à la diffusion de documents scientifiques de niveau recherche, publiés ou non, émanant des établissements d'enseignement et de recherche français ou étrangers, des laboratoires publics ou privés. 


\title{
A LEMKE-LIKE ALGORITHM FOR THE MULTICLASS NETWORK EQUILIBRIUM PROBLEM
}

\author{
FRÉDÉRIC MEUNIER AND THOMAS PRADEAU
}

\begin{abstract}
We consider a nonatomic congestion game on a connected graph, with several classes of players. Each player wants to go from its origin vertex to its destination vertex at the minimum cost and all players of a given class share the same characteristics: cost functions on each arc, and origin-destination pair. Under some mild conditions, it is known that a Nash equilibrium exists, but the computation of an equilibrium in the multiclass case is an open problem for general functions. We consider the specific case where the cost functions are affine and propose an extension of Lemke's algorithm able to solve this problem. At the same time, it provides a constructive proof of the existence of an equilibrium in this case.
\end{abstract}

\section{INTRODUCTION}

Context. Being able to predict the impact of a new infrastructure on the traffic in a transportation network is an old but still important objective for transport planners. In 1952, Wardrop [23] noted that after some while the traffic arranges itself to form an equilibrium and formalized principles characterizing this equilibrium. With the terminology of game theory, the equilibrium is a Nash equilibrium for a congestion game with nonatomic players. In 1956, Beckmann et al. [4] translated these principles as a mathematical program which turned out to be convex, opening the door to the tools from convex optimization. The currently most commonly used algorithm for such convex programs is probably the Frank-Wolfe algorithm (Frank and Wolfe [11]), because of its simplicity and its efficiency, but many other algorithms with excellent behaviors have been proposed, designed, and experimented.

One of the main assumptions used by Beckman to derive his program is the fact that all users are equally impacted by congestion. With the transportation terminology, it means that there is only one class. In order to improve the prediction of traffic patterns, researchers started in the 70s to study the multiclass situation where each class has its own way of being impacted by the congestion. Each class models a distinct mode of transportation, such as cars, trucks, or motorbikes. Dafermos [7, 8] and Smith [22] are probably the first who proposed a mathematical formulation of the equilibrium problem in the multiclass case. However, even if this problem has been the topic of many research works, an efficient algorithm for solving it remains to be designed, except in some special cases (Florian [9], Harker [12], Mahmassani and Mouskos [15], Marcotte and Wynter [16]). In particular, there is no general algorithm in the literature for solving the problem when the cost of each arc is in an affine dependence with the flow on it.

Our main purpose is to propose such an algorithm.

Model. We are given a directed graph $D=(V, A)$ modeling the transportation network. A route is an $s$ - $t$ path of $D$ and is called an $s$ - $t$ route. The set of all routes (resp. $s$ - $t$ routes) is denoted by $\mathcal{R}$ (resp. $\mathcal{R}_{(s, t)}$ ). The population of players is modeled as a bounded real interval $I$ endowed with the Lebesgue measure $\lambda$, the population measure. The set $I$ is partitioned into a finite number of measurable subsets $\left(I^{k}\right)_{k \in K}$ - the classes - modeling the players with same characteristics:

Key words and phrases. affine cost functions; congestion externalities; constructive proof; Lemke algorithm; nonatomic games; transportation network. 
they share a same collection of cost functions $\left(c_{a}^{k}: \mathbb{R}_{+} \rightarrow \mathbb{R}_{+}\right)_{a \in A}$, a same origin $s^{k}$, and a same destination $t^{k}$. A player in $I^{k}$ is said to be of class $k$. The set of vertices (resp. arcs) reachable from $s^{k}$ is denoted $V^{k}$ (resp. $A^{k}$ ).

A strategy profile is a measurable mapping $\sigma: I \rightarrow \mathcal{R}$ such that $\sigma(i) \in \mathcal{R}_{\left(s^{k}, t^{k}\right)}$ for all $k \in K$ and all $i \in I^{k}$. For each arc $a \in A$, the measure $x_{a}^{k}$ of the set of all class $k$ players $i$ such that $a$ is in $\sigma(i)$ is the class $k$ flow on $a$ in $\sigma$ :

$$
x_{a}^{k}=\lambda\left\{i \in I^{k}: a \in \sigma(i)\right\} .
$$

The total flow on $a$ is $x_{a}=\sum_{k \in K} x_{a}^{k}$. The cost of arc $a$ for a class $k$ player is then $c_{a}^{k}\left(x_{a}\right)$. For a class $k$ player, the cost of a route $r$ is defined as the sum of the costs of the arcs contained in $r$. Each player wants to select a minimum-cost route.

A strategy profile is a (pure) Nash equilibrium if each route is only chosen by players for whom it is a minimum-cost route. In other words, a strategy profile $\sigma$ is a Nash equilibrium if for each class $k \in K$ and each player $i \in I^{k}$ we have

$$
\sum_{a \in \sigma(i)} c_{a}^{k}\left(x_{a}\right)=\min _{r \in \mathcal{R}_{\left(s^{k}, t^{k}\right)}} \sum_{a \in r} c_{a}^{k}\left(x_{a}\right) .
$$

This game enters in the category of nonatomic congestion games with player-specific cost functions, see Milchtaich [17]. The problem of finding a Nash equilibrium for such a game is called the Multiclass Network Equilibrium Problem.

Contribution. Our results concern the case when the cost functions are affine and stricly increasing: for all $k \in K$ and $a \in A^{k}$, there exist $\alpha_{a}^{k}>0$ and $\beta_{a}^{k} \geq 0$ such that $c_{a}^{k}(x)=\alpha_{a}^{k} x+\beta_{a}^{k}$ for all $x \in \mathbb{R}_{+}$. In this case, the Multiclass Network Equilibrium Problem can be written as a linear complementarity problem. In 1965, Lemke [14] designed a pivoting algorithm for solving a linear complementarity problem under a quite general form. This algorithm has been adapted and extended several times - see for instance Adler and Verma [1], Asmuth et al. [3], Cottle et al. [6], Schiro et al. [20] to be able to deal with linear complementarity problems that do not directly fit in the required framework of the original Lemke algorithm.

We show that there exists a pivoting Lemke-like algorithm solving the Multiclass Network Equilibrium Problem when the costs are affine. To our knowledge, it is the first algorithm solving this problem. We prove its efficiency through computational experiments. Moreover, the algorithm provides the first constructive proof of the existence of an equilibrium for this problem. The initial proof of the existence from Schmeidler [21] uses a non-constructive approach with the help of a general fixed point theorem.

On our track, we extend slightly the notion of basis used in linear programming and linear complementarity programming to deal directly with unsigned variables, i.e. with equality constraints (by duality). Even if it is natural, we are not aware of previous use of such an approach.

Related works. We already gave some references of works related to ours with respect to the linear complementarity. The work by Schiro et al. [20] is one of them and deals actually with a more general problem as ours. However, the termination criterion for their algorithm is quite technical and it does not seem to be possible to prove that their algorithm terminates for sure on a equilibrium. Moreover, their algorithm is not a pure pivoting algorithm: some steps of the algorithm must be saved and restarts may be necessary; the matrices used in the pivot may have changing sizes. Finally, they do not treat equality constraints. Equality constraints can be encoded as inequality constraints, but they induce then additional dual variables, while in our approach, we are able to deal directly with them. 
Papers dealing with algorithms for solving the Multiclass Network Equilibrium Problems propose in general a Gauss-Seidel type diagonalization method, which consists in sequentially fixing the flows for all classes but one and solving the resulting single-class problem by methods of convex programming, see Florian [9], Harker [12], Mahmassani and Mouskos [15] for instance. In general, there is no simple condition ensuring the convergence to an equilibrium, even if there are sometimes local criterion (Florian and Spiess [10]). Another approach is proposed by Marcotte and Wynter [16]. For cost functions satisfying the "nested monotonicity" condition - a notion developed by Cohen and Chaplais [5] - they design a descent method for which they are able to prove the convergence to a solution of the problem. However, we were not able to find any paper with an algorithm solving the problem when the costs are polynomial functions, or even affine functions.

Structure of the paper. In Section 2, we explain how to write the Multiclass Network Equilibrium Problem as a linear complementarity problem. We get the formulation $(A M N E P(\boldsymbol{e}))$ on which the remaining of the paper focuses. Section 3 presents the notions that underly the Lemke-like algorithm. All these notions, likes basis, secondary ray, pivot, and so on, are classical in the context of the Lemke algorithm. They require however to be redefined in order to be able to deal with the features of $(A M N E P(\boldsymbol{e}))$. The algorithm is then described in Section 4. We also explain why it provides a constructive proof of the existence of an equilibrium. Section 5 is devoted to the experiments and shows the efficiency of the proposed approach.

\section{Formulation as a linear COMPlementarity PROBlem}

In this section, we formulate the Multiclass Network Equilibrium Problem as a complementarity problem which turns out to be linear when the cost functions are affine.

From now on, we assume that the cost functions are increasing. In the single-class case, i.e. $|K|=$ 1, the equilibrium flows are optimal solutions of a convex optimization problem, see Beckmann et al. [4]. If the flows $\boldsymbol{x}^{k^{\prime}}$ for $k^{\prime} \neq k$ are fixed, finding the equilibrium flows for the class $k$ is again a single-class problem which can be formulated as a convex optimization problem. With the help of the Karush-Kuhn-Tucker conditions, we get that the equilibrium flows $\left(x_{a}^{k}\right)$ coincide with the solutions of a system of the following form, where $\boldsymbol{b}=\left(b_{v}^{k}\right)$ is a given vector with $\sum_{v \in V^{k}} b_{v}^{k}=0$ for all $k$.

$\begin{array}{cr}\sum_{a \in \delta^{+}(v)} x_{a}^{k}=\sum_{a \in \delta^{-}(v)} x_{a}^{k}+b_{v}^{k} & k \in K, v \in V^{k} \\ c_{u v}^{k}\left(x_{u v}\right)+\pi_{u}^{k}-\pi_{v}^{k}-\mu_{u v}^{k}=0 & k \in K,(u, v) \in A^{k} \\ x_{a}^{k} \mu_{a}^{k}=0 & k \in K, a \in A^{k} \\ x_{a}^{k} \geq 0, \mu_{a}^{k} \geq 0, \pi_{v}^{k} \in \mathbb{R} \quad k \in K, a \in A^{k}, v \in V^{k} .\end{array}$

Actually in our model, we should have moreover $b_{v}^{k}=0$ for $v \notin\left\{s^{k}, t^{k}\right\}$, and the inequalities $b_{s^{k}}^{k}>0$ and $b_{t^{k}}^{k}<0$, but we relax this condition to deal with a slightly more general problem. Moreover, in this more general form, we can easily require the problem to be non-degenerate, see Section 3.2.

Finding solutions for such systems is a complementarity problem, the word "complementarity" coming from the condition $x_{a}^{k} \mu_{a}^{k}=0$ for all $(a, k)$ such that $a \in A^{k}$.

We have thus the following proposition.

Proposition 1. $\left(\boldsymbol{x}^{k}\right)_{k \in K}$ is an equilibrium flow if and only if there exist $\boldsymbol{\mu}^{k} \in \mathbb{R}_{+}^{A^{k}}$ and $\boldsymbol{\pi}^{k} \in \mathbb{R}^{V^{k}}$ for all $k$ such that $\left(\boldsymbol{x}^{k}, \boldsymbol{\mu}^{k}, \boldsymbol{\pi}^{k}\right)_{k \in K}$ is a solution of the complementarity problem $\left(M N E P_{\text {gen }}\right)$. 
When the cost functions are affine $c_{a}^{k}(x)=\alpha_{a}^{k} x+\beta_{a}^{k}$, solving the Multiclass Network Equilibrium Problem amounts thus to solve the following linear complementarity problem

$$
\begin{array}{lr}
\sum_{a \in \delta^{+}(v)} x_{a}^{k}=\sum_{a \in \delta^{-}(v)} x_{a}^{k}+b_{v}^{k} & k \in K, v \in V^{k} \\
\alpha_{u v}^{k} \sum_{k^{\prime} \in K} x_{u v}^{k^{\prime}}+\pi_{u}^{k}-\pi_{v}^{k}-\mu_{u v}^{k}=-\beta_{u v}^{k} & k \in K,(u, v) \in A^{k} \\
x_{a}^{k} \mu_{a}^{k}=0 & k \in K, a \in A^{k} \\
x_{a}^{k} \geq 0, \mu_{a}^{k} \geq 0, \pi_{v}^{k} \in \mathbb{R} & k \in K, a \in A^{k}, v \in V^{k} .
\end{array}
$$

Similarly as for the Lemke algorithm, we rewrite the problem as an optimization problem. It will be convenient for the exposure of the algorithm, see Section 3. This problem is called the Augmented Multiclass Network Equilibrium Problem. It uses a vector $\boldsymbol{e}=\left(e_{a}^{k}\right)$ defined for all $k \in K$ and $a \in A^{k}$. Problem $(A M N E P(e))$ is

$(A M N E P(e))$

$$
\begin{aligned}
& \min \omega \\
& \text { s.t. } \sum_{a \in \delta^{+}(v)} x_{a}^{k}=\sum_{a \in \delta^{-}(v)} x_{a}^{k}+b_{v}^{k} \quad k \in K, v \in V^{k} \\
& \alpha_{u v}^{k} \sum_{k^{\prime} \in K} x_{u v}^{k^{\prime}}+\pi_{u}^{k}-\pi_{v}^{k}-\mu_{u v}^{k}+e_{u v}^{k} \omega=-\beta_{u v}^{k} \quad k \in K,(u, v) \in A^{k} \\
& x_{a}^{k} \mu_{a}^{k}=0 \quad k \in K, a \in A^{k} \\
& x_{a}^{k} \geq 0, \mu_{a}^{k} \geq 0, \omega \geq 0, \pi_{v}^{k} \in \mathbb{R} \quad k \in K, a \in A^{k}, v \in V^{k} .
\end{aligned}
$$

Some choices of $\boldsymbol{e}$ allow to find easily feasible solutions to problem $(A M N E P(\boldsymbol{e}))$. In Section $3, \boldsymbol{e}$ will be chosen in such a way. A key remark is that solving $(M N E P)$ amounts to find an optimal solution for $(A M N E P(\boldsymbol{e}))$ with $\omega=0$.

Without loss of generality, we impose that $\pi_{s^{k}}^{k}=0$ for all $k \in K$. It allows to rewrite problem $(A M N E P(\boldsymbol{e}))$ under the form

$$
\begin{array}{ll}
\min & \omega \\
\text { s.t. } & \bar{M}^{e}\left(\begin{array}{c}
\boldsymbol{x} \\
\boldsymbol{\mu} \\
\omega
\end{array}\right)+\left(\begin{array}{c}
\mathbf{0} \\
M^{T}
\end{array}\right) \boldsymbol{\pi}=\left(\begin{array}{c}
\boldsymbol{b} \\
-\boldsymbol{\beta}
\end{array}\right) \\
& \boldsymbol{x} \cdot \boldsymbol{\mu}=0 \\
& \boldsymbol{x} \geq \mathbf{0}, \boldsymbol{\mu} \geq \mathbf{0}, \omega \geq 0, \boldsymbol{\pi} \in \mathbb{R}^{\sum_{k} V^{k} \backslash\left\{s^{k}\right\}},
\end{array}
$$

where $\bar{M}^{e}$ and $C$ are defined as follows. (The matrix $\bar{M}^{e}$ is denoted with a superscript $\boldsymbol{e}$ in order to emphasize its dependency on $\boldsymbol{e})$.

We define $M=\operatorname{diag}\left(\left(M^{k}\right)_{k \in K}\right)$ where $M^{k}$ is the incidence matrix of the directed graph $\left(V^{k}, A^{k}\right)$ from which the $s^{k}$-row has been removed:

$$
M_{v, a}^{k}= \begin{cases}1 & \text { if } a \in \delta^{+}(v) \\ -1 & \text { if } a \in \delta^{-}(v) \\ 0 & \text { otherwise }\end{cases}
$$


We also define $C^{k}=\operatorname{diag}\left(\left(\alpha_{a}^{k}\right)_{a \in A^{k}}\right)$ for $k \in K$, and then $C$ the real matrix $\left.C=(\underbrace{\left(C^{k}, \cdots, C^{k}\right.}_{|K| \text { times }})_{k \in K}\right)$. Then let

$$
\bar{M}^{e}=\left(\begin{array}{ccc}
M & 0 & 0 \\
C & -I & e
\end{array}\right)
$$

For $k \in K$, the matrix $M^{k}$ has $\left|V^{k}\right|-1$ rows and $\left|A^{k}\right|$ columns, while $C^{k}$ is a square matrix with $\left|A^{k}\right|$ rows and columns. Then the whole matrix $\bar{M}^{e}$ has $\sum_{k \in K}\left(\left|A^{k}\right|+\left|V^{k}\right|-1\right)$ rows and $2\left(\sum_{k \in K}\left|A^{k}\right|\right)+1$ columns.

\section{BASEs, PIVOTS, AND RAYS}

3.1. Bases. We define $\mathcal{X}$ and $\mathcal{M}$ to be two disjoint copies of $\left\{(a, k): k \in K, a \in A^{k}\right\}$. We denote by $\phi^{x}(a, k)\left(\right.$ resp. $\left.\phi^{\mu}(a, k)\right)$ the element of $\mathcal{X}$ (resp. $\left.\mathcal{M}\right)$ corresponding to $(a, k)$. The set $\mathcal{X}$ models the set of all possible indices for the ' $x$ ' variables and $\mathcal{M}$ the set of all possible indices for the ' $\mu$ ' variables for problem $(A M N E P(\boldsymbol{e}))$. We consider moreover a dummy element $o$ as the index for the ' $\omega$ ' variable.

We define a basis for problem $(A M N E P(\boldsymbol{e}))$ to be a subset $B$ of the set $\mathcal{X} \cup \mathcal{M} \cup\{o\}$ such that the square matrix of size $\sum_{k \in K}\left(\left|A^{k}\right|+\left|V^{k}\right|-1\right)$ defined by

$$
\left(\begin{array}{c|c}
\bar{M}_{B}^{e} & \mathbf{0} \\
M^{T}
\end{array}\right)
$$

is nonsingular. Note that this definition is not standard. In general, a basis is defined in this way but without the submatrix $\left(\begin{array}{c}0 \\ M^{T}\end{array}\right)$ corresponding to the ' $\pi$ ' columns. We use this definition in order to be able to deal directly with the unsigned variables ' $\pi$ '. We will see that this approach is natural (and could be used for linear programming as well). However, we are not aware of a previous use of such an approach.

As a consequence of this definition, since $M^{T}$ has $\sum_{k \in K}\left(\left|V^{k}\right|-1\right)$ columns, a basis is always of cardinality $\sum_{k \in K}\left|A^{k}\right|$.

Remark 1. In particular, since the matrix is nonsingular and since $M^{T}$ has $\sum_{k \in K}\left|A^{k}\right|$ rows, the first $\sum_{k \in K}\left(\left|V^{k}\right|-1\right)$ rows of $\bar{M}_{B}^{e}$ have each a nonzero entry. This property is used below, especially in the proof of Lemma 4 .

The following additional notation is useful: given a subset $Z \subseteq \mathcal{X} \cup \mathcal{M} \cup\{o\}$, we denote by $Z^{x}$ the set $\left(\phi^{x}\right)^{-1}(Z \cap \mathcal{X})$ and by $Z^{\mu}$ the set $\left(\phi^{\mu}\right)^{-1}(Z \cap \mathcal{M})$. In other words, $(a, k)$ is in $Z^{x}$ if and only if $\phi^{x}(a, k)$ is in $Z$, and similarly for $Z^{\mu}$.

3.2. Basic solutions and non-degeneracy. Let $B$ a basis. If it contains $o$, the unique solution $(\overline{\boldsymbol{x}}, \overline{\boldsymbol{\mu}}, \bar{\omega}, \overline{\boldsymbol{\pi}})$ of

$$
\left\{\begin{array}{c|c}
\left(\begin{array}{c|c}
\bar{M}_{B}^{e} & \mathbf{0} \\
M^{T}
\end{array}\right)\left(\begin{array}{c}
\boldsymbol{x}_{B^{x}} \\
\boldsymbol{\mu}_{B^{\mu}} \\
\omega \\
\boldsymbol{\pi}
\end{array}\right)=\left(\begin{array}{c}
\boldsymbol{b} \\
-\boldsymbol{\beta}
\end{array}\right) \\
x_{a}^{k}=0 \quad \text { for all }(a, k) \notin B^{x} \\
\mu_{a}^{k}=0 \quad \text { for all }(a, k) \notin B^{\mu} .
\end{array}\right.
$$

is called the basic solution associated to $B$. 
If $B$ does not contain $o$, we define similarly its associated basic solution. It is the unique solution $(\overline{\boldsymbol{x}}, \overline{\boldsymbol{\mu}}, \bar{\omega}, \overline{\boldsymbol{\pi}})$ of

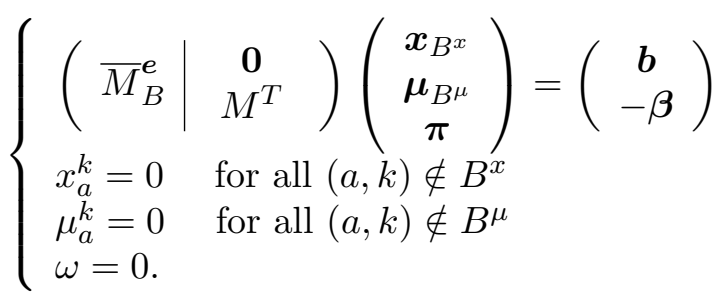

A basis is said to be feasible if the associated basic solution is such that $\overline{\boldsymbol{x}}, \overline{\boldsymbol{\mu}}, \bar{\omega} \geq 0$.

The problem $(A M N E P(\boldsymbol{e}))$ is said to satisfy the non-degeneracy assumption if, for any feasible basis $B$, the associated basic solution $(\overline{\boldsymbol{x}}, \overline{\boldsymbol{\mu}}, \bar{\omega}, \overline{\boldsymbol{\pi}})$ is such that

$$
\left((a, k) \in B^{x} \Rightarrow \bar{x}_{a}^{k}>0\right) \text { and }\left((a, k) \in B^{\mu} \Rightarrow \bar{\mu}_{a}^{k}>0\right) .
$$

Note that if we had defined the vector $\boldsymbol{b}$ to be 0 on all vertices $v \notin\left\{s^{k}, t^{k}\right\}$, the problem would not in general satisfy the non-degeneracy assumption. An example of a basis for which the condition fails to be satisfied is the basis $B^{i n i}$ defined in Section 3.5. Remark 3 in that section details the example.

3.3. Pivots and polytope. The following lemmas are key results that will eventually lead to the Lemke-like algorithm. They are classical for the usual definition of bases. Since we have extended the definition, we have to prove that they still hold.

Lemma 1. Let $B$ be a feasible basis for problem $(A M N E P(\boldsymbol{e}))$ and assume non-degeneracy. Let $i$ be an index in $\mathcal{X} \cup \mathcal{M} \cup\{o\} \backslash B$. Then there is at most one feasible basis $B^{\prime} \neq B$ in the set $B \cup\{i\}$.

The operation consisting in computing $B^{\prime}$ given $B$ and the entering index $i$ is called the pivot operation.

Proof of Lemma 1. See Appendix.

If we are able to determine an index in $\mathcal{X} \cup \mathcal{M} \cup\{o\} \backslash B$ for any basis $B$, Lemma 1 leads to a "pivoting" algorithm. At each step, we have a current basis $B^{\text {curr }}$, we determine the entering index $i$, and we compute the new basis in $B^{\text {curr }} \cup\{i\}$, if it exists, which becomes the new current basis $B^{\text {curr }}$; and so on. Next lemma allows to characterize situations where there is no new basis, i.e. situations for which the algorithm gets stuck.

The feasible solutions of $(A M N E P(\boldsymbol{e}))$ belong to the polytope

$$
\begin{array}{r}
\mathcal{P}(\boldsymbol{e})=\left\{(\boldsymbol{x}, \boldsymbol{\mu}, \omega, \boldsymbol{\pi}): \bar{M}^{e}\left(\begin{array}{l}
\boldsymbol{x} \\
\boldsymbol{\mu} \\
\omega
\end{array}\right)+\left(\begin{array}{c}
\mathbf{0} \\
M^{T}
\end{array}\right) \boldsymbol{\pi}=\left(\begin{array}{c}
\boldsymbol{b} \\
-\boldsymbol{\beta}
\end{array}\right),\right. \\
\left.\boldsymbol{x} \geq \mathbf{0}, \boldsymbol{\mu} \geq \mathbf{0}, \boldsymbol{\pi} \geq \mathbf{0}, \omega \in \mathbb{R}_{+}\right\}
\end{array}
$$

Lemma 2. Let $B$ be a feasible basis for problem $(A M N E P(\boldsymbol{e}))$ and assume non-degeneracy. Let $i$ be an index in $\mathcal{X} \cup \mathcal{M} \cup\{o\} \backslash B$. If there is no feasible basis $B^{\prime} \neq B$ in the set $B \cup\{i\}$, then the polytope $\mathcal{P}(\boldsymbol{e})$ contains an infinite ray originating at the basic solution associated to $B$.

Proof. See Appendix. 
3.4. Complementarity and twin indices. A basis $B$ is said to be complementary if for every $(a, k)$ with $a \in A^{k}$, we have $(a, k) \notin B^{x}$ or $(a, k) \notin B^{\mu}$ : for each $(a, k)$, one of the components $x_{a}^{k}$ or $\mu_{a}^{k}$ is not activated in the basic solution. In case of non-degeneracy, it coincides with the condition $\boldsymbol{x} \cdot \boldsymbol{\mu}=0$. An important point to be noted for a complementary basis $B$ is that if $o \in B$, then there is $\left(a_{0}, k_{0}\right)$ with $a_{0} \in A^{k_{0}}$ such that

- $\left(a_{0}, k_{0}\right) \notin B^{x}$ and $\left(a_{0}, k_{0}\right) \notin B^{\mu}$, and

- for all $(a, k) \neq\left(a_{0}, k_{0}\right)$ with $a \in A^{k}$, exactly one of the relations $(a, k) \in B^{x}$ and $(a, k) \in B^{\mu}$ is satisfied.

This is a direct consequence of the fact that there are exactly $\sum_{k \in K}\left|A^{k}\right|$ elements in a basis and that each $(a, k)$ is not present in at least one of $B^{x}$ and $B^{\mu}$. In case of non-degeneracy, this point amounts to say that $x_{a}^{k}=0$ or $\mu_{a}^{k}=0$ for all $(a, k)$ with $a \in A^{k}$ and that there is exactly one such pair, denoted $\left(a_{0}, k_{0}\right)$, such that both are equal to 0 .

We say that $\phi^{x}\left(a_{0}, k_{0}\right)$ and $\phi^{\mu}\left(a_{0}, k_{0}\right)$ for such $\left(a_{0}, k_{0}\right)$ are the twin indices.

3.5. Initial feasible basis. An $s$-arborescence in a directed graph in a spanning tree rooted at $s$ that has a directed path from $s$ to any vertex of the graph. We define a collection $\mathcal{T}=\left(T^{k}\right)_{k \in K}$ where $T^{k} \subseteq A^{k}$ is an $s^{k}$-arborescence of $\left(V^{k}, A^{k}\right)$. Then the vector $\boldsymbol{e}=\left(e_{a}^{k}\right)_{k \in K}$ is chosen with the help of $\mathcal{T}$ by

$$
e_{a}^{k}= \begin{cases}1 & \text { if } a \notin T^{k} \\ 0 & \text { otherwise }\end{cases}
$$

With this choice of $\boldsymbol{e}$ in problem $(A M N E P(\boldsymbol{e}))$, there is an initial feasible complementary basis easily computable. The remaining of this subsection is devoted to the computation of such an initial basis.

Let the set of indices $Y \subseteq \mathcal{X} \cup \mathcal{M} \cup\{o\}$ be defined by

$$
Y=\left\{\phi^{x}(a, k): a \in T^{k}, k \in K\right\} \cup\left\{\phi^{\mu}(a, k): a \in A^{k} \backslash T^{k}, k \in K\right\} \cup\{o\} .
$$

The subset $Y$ has cardinality $\sum_{k \in K}\left|A^{k}\right|+1$. We are going to show that $Y$ contains a feasible complementary basis. We proceed by studying the solutions of the system

$$
\left\{\begin{array}{c|c}
\left(\begin{array}{c}
\bar{M}_{Y}^{e} \\
M^{T}
\end{array}\right)\left(\begin{array}{c}
\boldsymbol{x}_{Y^{x}} \\
\boldsymbol{\mu}_{Y^{\mu}} \\
\omega \\
\boldsymbol{\pi}
\end{array}\right)=\left(\begin{array}{c}
\boldsymbol{b} \\
-\boldsymbol{\beta}
\end{array}\right) \\
x_{a}^{k}=0 \quad \text { for all }(a, k) \notin Y^{x} \\
\mu_{a}^{k}=0 \quad \text { for all }(a, k) \notin Y^{\mu} .
\end{array}\right.
$$

The matrix $M_{T^{k}}^{k}$ is nonsingular (see for instance the book by Ahuja et al. [2]). There is thus a unique solution $\boldsymbol{x}$ to system $\left(S^{e}\right)$. Since for $a \in T^{k}$ we have $e_{a}^{k}=0$ and $\phi^{\mu}(a, k) \notin Y$, any solution of system $\left(S^{e}\right)$ satisfies the equalities

$$
\alpha_{u v}^{k} \sum_{k^{\prime} \in K} x_{u v}^{k^{\prime}}+\pi_{u}^{k}-\pi_{v}^{k}=-\beta_{u v}^{k}, \quad \text { for all } k \in K \text { and }(u, v) \in T^{k} .
$$

Recall that we defined $\pi_{s^{k}}^{k}=0$. Since $T^{k}$ is a spanning tree of $\left(V^{k}, A^{k}\right)$ for all $k$, these equations completely determine the values of the $\pi_{v}^{k}$.

Moreover, since for $a \notin T^{k}$ we have $e_{a}^{k}=1$ and $\phi^{x}(a, k) \notin Y$, any solution of system $\left(S^{e}\right)$ satisfies the equalities

$$
-\mu_{u v}^{k}+\omega+\pi_{u}^{k}-\pi_{v}^{k}=-\beta_{u v}^{k}, \quad \text { for all } k \in K \text { and }(u, v) \notin T^{k} .
$$


If $\beta_{u v}^{k}+\pi_{u}^{k}-\pi_{v}^{k} \geq 0$ for all $k \in K$ and $(u, v) \notin T^{k}$, then we have an optimal solution of problem $(A M N E P(\boldsymbol{e}))$ with $\omega=0$, and we have solved our problem. We can thus assume that $\beta_{u v}^{k}+\pi_{u}^{k}-\pi_{v}^{k}<0$ for at least one triple $u, v, k$. Let $u_{0}, v_{0}, k_{0}$ be such a triple minimizing $\beta_{u v}^{k}+\pi_{u}^{k}-\pi_{v}^{k}$ and let $a_{0}=\left(u_{0}, v_{0}\right)$. Note that Equation (5) implies that

$$
\mu_{u v}^{k} \geq \mu_{u_{0} v_{0}}^{k_{0}}, \quad \text { for all } k \in K \text { and }(u, v) \notin T^{k} .
$$

Lemma 3. The subset $B^{i n i} \subseteq \mathcal{X} \cup \mathcal{M} \cup\{o\}$ defined by $B^{i n i}=Y \backslash\left\{\phi^{\mu}\left(a_{0}, k_{0}\right)\right\}$ is a feasible complementary basis for problem $(A M N E P(\boldsymbol{e}))$.

We emphasize that $B^{\text {ini }}$ depends on the collection $\mathcal{T}$ of arborescences.

Proof of Lemma 3. For such a $B^{i n i}$, system (2) has a unique solution. The proof is exactly the one given just above: first we see that $\boldsymbol{x}$ is uniquely determined and then $\boldsymbol{\pi}$. Then by definition of $\left(a_{0}, k_{0}\right)$, since $\phi^{\mu}\left(a_{0}, k_{0}\right)$ is not in $B^{i n i}$, we must have

$$
\mu_{u_{0} v_{0}}^{k_{0}}=0 \quad \text { and } \quad \omega=-\beta_{u_{0} v_{0}}^{k_{0}}-\pi_{u_{0}}^{k_{0}}+\pi_{v_{0}}^{k_{0}} .
$$

Finally, Equation (5) determines the values of the $\mu_{u v}^{k}$ for $k \in K$ and $(u, v) \notin T^{k}$, and Equation (6) ensures that these values are nonnegative. Therefore, $B^{i n i}$ is a basis, and it is feasible because all $x_{a}^{k}$ and $\mu_{a}^{k}$ in the solution are nonnegative. Furthermore, for each $(a, k)$ with $a \in A^{k}$, at least one of $\phi^{x}(a, k)$ and $\phi^{\mu}(a, k)$ is not in $B^{i n i}$.

Hence, the subset $B^{i n i}$ is a feasible complementary basis.

Note that the basis $B^{i n i}$ is polynomially computable.

Remark 2. A short examination of the proof makes clear that the following claim is true: Assuming non-degeneracy, if $B$ is a feasible basis such that $B^{x}=\left\{(a, k): a \in T^{k}, k \in K\right\}$, then $B=B^{i n i}$. The fact that the $T^{k}$ are arborescences fixes completely $\boldsymbol{x}$, and then $\boldsymbol{\pi}$. The fact that $B$ is a feasible basis forces $\omega$ to be equal to the maximal value of $-\beta_{u v}^{k}-\pi_{u}^{k}+\pi_{v}^{k}$ (except of course if this value is nonpositive, in which case we have already solved our problem), which in turn fixes the values of the $\mu_{u v}^{k}$.

Remark 3. As already announced in Section 3.2, if we had defined the vector $\boldsymbol{b}$ to be 0 on all vertices $v \notin\left\{s^{k}, t^{k}\right\}$, the problem would not satisfy the non-degeneracy assumption as soon as there is $k \in K$ such that $T^{k}$ has a vertex of degree 3 (which happens when $\left(V^{k}, A^{k}\right)$ has no Hamiltonian path). In this case, the basis $B^{\text {ini }}$ shows that the problem is degenerate. Since the unique solution $\boldsymbol{x}^{k}$ of $M_{T^{k}}^{k} \boldsymbol{x}^{k}=\boldsymbol{b}^{k}$ consists in sending the whole demand on the unique route in $T^{k}$ from $s^{k}$ to $t^{k}$, we have for all arcs $a \in T^{k}$ not belonging to this route $x_{a}^{k}=0$ while $(a, k) \in B^{\text {ini,x }}$.

3.6. No secondary ray. Let $\left(\overline{\boldsymbol{x}}^{i n i}, \overline{\boldsymbol{\mu}}^{i n i}, \bar{\omega}^{i n i}, \overline{\boldsymbol{\pi}}^{i n i}\right)$ be the feasible basic solution associated to the initial basis $B^{i n i}$, computed according to Lemma 3 and with $\boldsymbol{e}$ given by Equation (4). The following inifinite ray

$$
\rho^{i n i}=\left\{\left(\overline{\boldsymbol{x}}^{i n i}, \overline{\boldsymbol{\mu}}^{i n i}, \bar{\omega}^{i n i}, \overline{\boldsymbol{\pi}}^{i n i}\right)+t(\mathbf{0}, \boldsymbol{e}, 1, \mathbf{0}): t \geq 0\right\},
$$

has all its points in $\mathcal{P}(\boldsymbol{e})$. This ray with direction $(\mathbf{0}, \boldsymbol{e}, 1, \mathbf{0})$ is called the primary ray. In the terminology of the Lemke algorithm, another infinite ray originating at a solution associated to a feasible complementary basis is called a secondary ray. System $(A M N E P(\boldsymbol{e}))$ has no secondary ray for the $\boldsymbol{e}$ chosen according to Equation (4) in Section 3.5.

Lemma 4. Let $\boldsymbol{e}$ be defined by Equation (4). Under the non-degeneracy assumption, there is no secondary ray in $\mathcal{P}(\boldsymbol{e})$.

Proof. See Appendix. 
3.7. A Lemke-like algorithm. Assuming non-degeneracy, the combination of Lemma 1 and the point explicited in Section 3.4 give raise to a Lemke-like algorithm. Two feasible complementary bases $B$ and $B^{\prime}$ are said to be neighbors if $B^{\prime}$ can be obtained from $B$ by a pivot operation using one of the twin indices as an entering index, see Section 3.4. Note that is is a symmetrical notion: $B$ can then also be obtained from $B^{\prime}$ by a similar pivot operation. The abstract graph whose vertices are the feasible complementary bases and whose edges connect neighbor bases is thus a collection of paths and cycles. In Section 3.5, we have seen that for the chosen vector $\boldsymbol{e}$, we can find in polynomial time an initial feasible complementary basis for $(A M N E P(\boldsymbol{e}))$. This initial basis has exactly one neighbor according to Lemma 2 since there is a primary ray and no secondary ray (Lemma 4).

Algorithm 1 explains how to follow the path starting at this initial feasible complementary basis. Function EnteringIndex $\left(B, i^{\prime}\right)$ is defined for a feasible complementary basis $B$ and an index $i^{\prime} \notin B$ being a twin index of $B$ and computes the other twin index $i \neq i^{\prime}$. Function Leaving $\operatorname{Index}(B, i)$ is defined for a feasible complementary basis $B$ and an index $i \notin B$ and computes the unique index $j \neq i$ such that $B \cup\{i\} \backslash\{j\}$ is a feasible complementary basis (see Lemma 1 ).

Since there is no secondary ray (Lemma 4), a pivot operation is possible because of Lemma 2 as long as there are twin indices. By finiteness, a component in the abstract graph having an endpoint necessarily has another endpoint. It implies that the algorithm reaches at some moment a basis $B$ without twin indices. Such a basis is such that $o \notin B$ (Section 3.4), which implies that we have a solution of problem $(A M N E P(\boldsymbol{e}))$ with $\omega=0$, i.e. a solution of problem $(M N E P)$, and thus a solution of our initial problem.

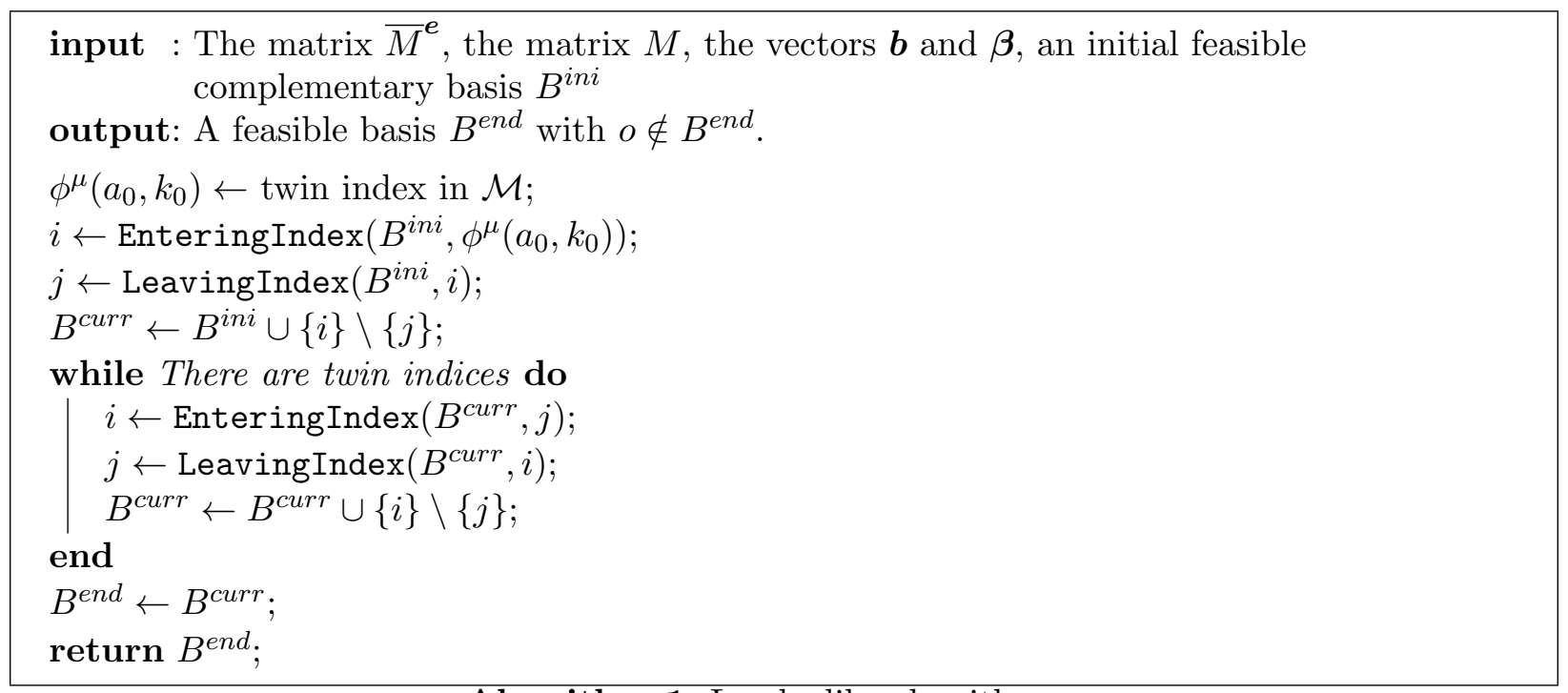

Algorithm 1: Lemke-like algorithm

\section{Algorithm And main Result}

We are now in a position to describe the full algorithm under the non-degeneracy assumption.

(1) For each $k \in K$, compute a collection $\mathcal{T}=\left(T^{k}\right)$ where $T^{k} \subseteq A^{k}$ is an $s^{k}$-arborescence of $\left(V^{k}, A^{k}\right)$.

(2) Define $\boldsymbol{e}$ as in Equation (4) (which depends on $\mathcal{T}$ ).

(3) Compute the unique solution $\left(\overline{\boldsymbol{x}}^{i n i}, \overline{\boldsymbol{\mu}}^{i n i}, \bar{\omega}^{i n i}, \overline{\boldsymbol{\pi}}^{i n i}\right)$ of system $\left(S^{\boldsymbol{e}}\right)$, with $Y=\left\{\phi^{x}(a, k)\right.$ : $\left.a \in T^{k}, k \in K\right\} \cup\left\{\phi^{\mu}(a, k): a \in A^{k} \backslash T^{k}, k \in K\right\} \cup\{o\}$. 
(4) If $\beta_{u v}^{k}+\bar{\pi}_{u}^{i n i, k}-\bar{\pi}_{v}^{i n i, k} \geq 0$ for all $u, v, k$ such that $(u, v) \in A^{k} \backslash T^{k}$, then we have a solution of problem $(M N E P)$, see Section 3.5.

(5) Otherwise, let $B^{i n i}$ be defined as in Lemma 3 and apply Algorithm 1, which returns a basis $B^{\text {end }}$.

(6) Compute the basic solution associated to $B^{\text {end }}$.

All the elements proved in Section 3 leads finally to the following result.

Theorem 1. Under the non-degeneracy assumption, this algorithm solves problem (MNEP), i.e. the Multiclass Network Equilibrium Problem with affine costs.

This result provides actually a constructive proof of the existence of an equilibrium for the Multiclass Network Equilibrium Problem when the cost are affine and strictly increasing, even if the non-degeneracy assumption is not satisfied. If we compute $\boldsymbol{b}=\left(b_{v}^{k}\right)$ strictly according to the model, we have

$$
b_{v}^{k}= \begin{cases}\lambda\left(I^{k}\right) & \text { if } v=s^{k} \\ -\lambda\left(I^{k}\right) & \text { if } v=t^{k} \\ 0 & \text { otherwise. }\end{cases}
$$

In this case, the non-degeneracy assumption is not satisfied as it has been noted at the end of Section 3.5 (Remark 3). Anyway, we can slightly perturb $\boldsymbol{b}$ and $-\boldsymbol{\beta}$ in such a way that any feasible complementary basis of the perturbated problem is still a feasible complementary basis for the original problem. Such a perturbation exists by standard arguments, see Cottle et al. [6]. Theorem 1 ensures then the termination of the algorithm on a feasible complementary basis $B$ whose basic solution is such that $\omega=0$. It provides thus a solution for the original problem.

It shows also that the problem of finding such an equilibrium belongs to the PPAD complexity class. The PPAD class - defined by Papadimitriou [19] in 1994 - is the complexity class of functional problems for which we know the existence of the object to be found because of a (oriented) pathfollowing argument. There are PPAD-complete problems, i.e. PPAD problems as hard as any problem in the PPAD class, see Kintali et al. [13] for examples of such problems. A natural question would be whether the Multiclass Network Equilibrium Problem with affine costs is PPAD-complete. We do not know the answer.

Another consequence of Theorem 1 is that if the demands $\lambda\left(I^{k}\right)$ and the cost parameters $\alpha_{a}^{k}, \beta_{a}^{k}$ are rational numbers, then there exists an equilibrium inducing rational flows on each arc and for each class $k$. It is reminiscent of a similar result for two players matrix games: if the matrices involve only rational entries, there is an equilibrium involving only rational numbers (Nash [18]).

\section{Computational experiments}

5.1. Instances. The experiments are made on $n \times n$ grid graphs. For each pair of adjacent vertices $u$ and $v$, both $\operatorname{arcs}(u, v)$ and $(v, u)$ are present. We built several instances on these graphs with various sizes $n$, various numbers of classes, and various cost parameters $\alpha_{a}^{k}, \beta_{a}^{k}$. The cost parameters are chosen such that for all $a$ and all $k$

$$
\alpha_{a}^{k} \in[1,10] \quad \text { and } \quad \beta_{a}^{k} \in[0,100] .
$$

5.2. Results. The algorithm has been coded in $\mathrm{C}++$ and tested on a PC Intel ${ }^{\circledR}$ Core ${ }^{\mathrm{TM}}$ i5-2520M clocked at $2.5 \mathrm{GHz}$, with $4 \mathrm{~GB}$ RAM. The experiments are currently in progress. However, some preliminary computational results are given in Table 1. Each row of the table contains average figures obtained on five instances on the same graph and with the same number classes, but with various origins, destinations, and costs parameters. 


\begin{tabular}{cc|cc|ccc} 
Grid & Classes & Vertices & Arcs & Pivots & $\begin{array}{c}\text { Algorithm 1 } \\
\text { (seconds) }\end{array}$ & $\begin{array}{c}\text { Inversion } \\
\text { (seconds) }\end{array}$ \\
\hline $2 \times 2$ & 50 & 4 & 8 & 52 & 0.4 & 4.5 \\
$2 \times 2$ & 100 & 4 & 8 & 114 & 3.5 & 34 \\
$2 \times 2$ & 200 & 4 & 8 & 234 & 29 & 267 \\
\hline $4 \times 4$ & 10 & 16 & 48 & 117 & 1.3 & 6.7 \\
$4 \times 4$ & 20 & 16 & 48 & 241 & 10 & 52 \\
$4 \times 4$ & 50 & 16 & 48 & 646 & 187 & 877 \\
\hline $8 \times 8$ & 2 & 64 & 224 & 119 & 1.0 & 5.0 \\
$8 \times 8$ & 5 & 64 & 224 & 288 & 16 & 80 \\
$8 \times 8$ & 10 & 64 & 224 & 663 & 143 & 616
\end{tabular}

TABLE 1. Performances of the complete algorithm for various instance sizes

The columns "Classes", "Vertices", and "Arcs" contain respectively the number of classes, the number of vertices, and the number of arcs. The column "Pivots" contains the number of pivots performed by the algorithm. They are done during Step 5 in the description of the algorithm in Section 4 (application of Algorithm 1). The column "Algorithm 1" provides the time needed for the whole execution of this pivoting step. The preparation of this pivoting step requires a first matrix inversion, and the final computation of the solution requires such an inversion as well. The times needed to perform these inversions are given in the column "Inversion". The total time needed by the complete algorithm to solve the problem is the sum of the "Algorithm 1" time and twice the "Inversion" time, the other steps of the algorithm taking a negligible time.

It seems that the number of pivots remains always reasonable. Even if the time needed to solve large instances is sometimes important with respect to the size of the graph, the essential computation time is spent on the two matrix inversions. The program has not been optimized. Since there are several efficient techniques known for inverting matrices, the results can be considered as very positive.

\section{REFERENCES}

[1] I. Adler and S. Verma. The linear complementarity problem, Lemke algorithm, perturbation, and the complexity class PPAD. Technical report, 2011.

[2] R.K. Ahuja, T.L. Magnanti, and J.B. Orlin. Network Flows: Theory, Algorithms, and Applications. Prentice-Hall.

[3] R. Asmuth, B.C. Eaves, and E.L. Peterson. Computing economic equilibria on affine networks with Lemke's algorithm. Math. Oper. Res., 4:209-214, 1979.

[4] M. Beckmann, C. B. McGuire, and C. B. Winsten. Studies in Economics of Transportation. Yale University Press, New Haven, CT, 1956.

[5] G. Cohen and F. Chaplais. Nested monotonicity for variational inequalities over product of spaces and convergence of iterative algorithms. J. Optim. Theory Appl., 59:369-390, 1988.

[6] R.W. Cottle, J.S. Pang, and R.E Stone. The linear complementarity problem. 1992.

[7] S. Dafermos. The traffic assignment problem for multiclass-user transportation networks. Transportation Sci., 6:73-87, 1972.

[8] S. Dafermos. Traffic equilibrium and variational inequalities. Transportation Sci., 14:42-54, 1980.

[9] M. Florian. A traffic equilibrium model of travel by car and public transit modes. Transportation Sci., 11:166-179, 1977. 
[10] M. Florian and H. Spiess. The convergence of diagonalisation algorithms for asymmetric network equilibrium problems. Transportation Res. part B, 16:477-483, 1982.

[11] M. Frank and P. Wolfe. An algorithm for quadratic programming. Naval Research Logistics Quarterly, 3:95-110, 1956.

[12] P.T. Harker. Accelerating the convergence of the diagonalization and projection algorithms for finite-dimensional variational inequalities. Math. Programming, 48:29-59, 1988.

[13] S. Kintali, Poplawski, L.J., R. Rajaraman, R. Sundaram, and S.-H. Teng. Reducibility among fractional stability problems. In 50th IEEE Symposium on Foundations of Computer Science (FOCS), Atlanta, 2009.

[14] C.E. Lemke. Bimatrix equilibrium points and equilibrium programming. Management Science, 11:681-689, 1965.

[15] H.S. Mahmassani and K.C. Mouskos. Some numerical results on the diagonalization algorithm for network assignment with asymmetric interactions between cars and trucks. Transportation Res. part B, 22:275-290, 1998.

[16] P. Marcotte and L. Wynter. A new look at the multiclass network equilibrium problem. Transportation Sci., 38:282-292, 2004.

[17] I. Milchtaich. Congestion games with player-specific payoff functions. Games Econom. Behavior, 13:111-124, 1996.

[18] J.F. Nash. Non-cooperative games. Annals of Mathematics, 54:286-295, 1951.

[19] C. Papadimitriou. On the complexity of the parity argument and other inefficient proofs of existence. Journal of Computer and System Sciences, 48:498-532, 1994.

[20] D.A. Schiro, J-S Pang, and U.V. Shanbhag. On the solution of affine generalized Nash equilibrium problems with shared constraints by Lemke's method. Math. Program., 2012.

[21] D. Schmeidler. Equilibrium points on nonatomic games. J. Statist. Phys., 7:295-300, 1970.

[22] M.J. Smith. The existence, uniqueness, and stability of traffic equilibria. Transportation Res. part B., 15:443-451, 1979.

[23] J. G. Wardrop. Some theoretical aspects of road traffic research. Proc. Inst. Civil Engineers, $2: 325-378,1952$.

\section{APPENDIX}

Proof of Lemma 1. Let $(\overline{\boldsymbol{x}}, \overline{\boldsymbol{\mu}}, \overline{\boldsymbol{\omega}}, \overline{\boldsymbol{\pi}})$ be the basic solution associated to $B$ and let $Y=B \cup\{i\}$. The set of solutions

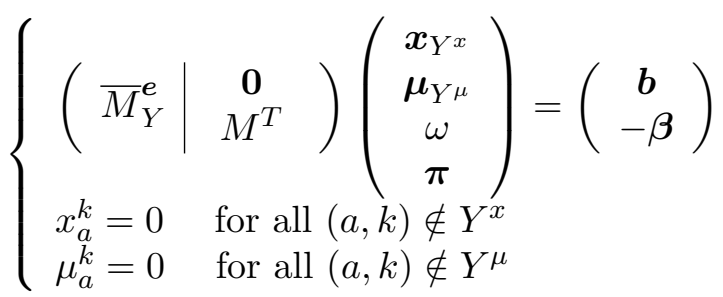

is a one-dimensional line in $\mathbb{R}^{1+\sum_{k \in K}\left(2\left|A^{k}\right|+\left|V^{K}\right|-1\right)}$ (the space of all variables) and passing through $(\overline{\boldsymbol{x}}, \overline{\boldsymbol{\mu}}, \overline{\boldsymbol{\omega}}, \overline{\boldsymbol{\pi}})$. The bases in $Y$ correspond to intersections of this line with the boundary of

$$
Q=\left\{(\boldsymbol{x}, \boldsymbol{\mu}, \omega, \boldsymbol{\pi}): x_{a}^{k} \geq 0, \mu_{a}^{k} \geq 0, \omega \geq 0, \text { for all } k \in K \text { and } a \in A^{k}\right\} .
$$

This latter set being convex (it is a polyhedron), the line intersect at most twice its boundary under the non-degeneracy assumption.

Proof of Lemma 2. The proof is similar as the one of Lemma 1, of which we take the same notions and notations. If $B$ is the only feasible basis, then the line intersects the boundary of $Q$ exactly once. Because of the non-degeneracy assumption, it implies that there is an infinite ray originating at $(\overline{\boldsymbol{x}}, \overline{\boldsymbol{\mu}}, \overline{\boldsymbol{\omega}}, \overline{\boldsymbol{\pi}})$ and whose points are all feasible. 
Proof of Lemma 4. Suppose that $\mathcal{P}(\boldsymbol{e})$ contains an infinite ray

$$
\rho=\left\{(\overline{\boldsymbol{x}}, \overline{\boldsymbol{\mu}}, \bar{\omega}, \overline{\boldsymbol{\pi}})+t\left(\boldsymbol{x}^{d i r}, \boldsymbol{\mu}^{d i r}, \omega^{d i r}, \boldsymbol{\pi}^{d i r}\right): t \geq 0\right\}
$$

where $(\overline{\boldsymbol{x}}, \overline{\boldsymbol{\mu}}, \bar{\omega}, \overline{\boldsymbol{\pi}})$ is a feasible complementary basic solution associated to a basis $B$.

We first show that $\boldsymbol{x}^{d i r}=0$. For a contradiction, suppose that it is not the case and let $k$ be such that $\boldsymbol{x}^{d i r, k}$ is not zero. Since the points of $\rho$ must satisfy the system $(A M N E P(\boldsymbol{e}))$ for all $t \geq 0$, we have that $\left(\boldsymbol{x}^{d i r}, \boldsymbol{\mu}^{\text {dir }}, \omega^{\text {dir }}, \boldsymbol{\pi}^{\text {dir }}\right)$ must satisfy for all $v \in V^{k}$

$$
\sum_{a \in \delta^{+}(v)} x_{a}^{d i r, k}=\sum_{a \in \delta^{-}(v)} x_{a}^{d i r, k}
$$

which shows that $\boldsymbol{x}^{k}$ is a circulation in the directed graph $\left(V^{k}, A^{k}\right)$. Moreover, we must have for all $(u, v) \in A^{k}$

$$
\alpha_{u v}^{k} \sum_{k^{\prime} \in K} x_{u v}^{d i r, k^{\prime}}+\pi_{u}^{d i r, k}-\pi_{v}^{d i r, k}-\mu_{u v}^{d i r, k}+e_{u v}^{k} \omega^{d i r}=0,
$$

where we have defined $\pi_{s^{k}}^{d i r, k}=0$. The following relations must also be satisfied:

$$
\boldsymbol{x}^{d i r} \cdot \boldsymbol{\mu}^{d i r}=0
$$

and

$$
\boldsymbol{x}^{\text {dir }} \geq \mathbf{0}, \boldsymbol{\mu}^{\text {dir }} \geq \mathbf{0}, \omega^{\text {dir }} \geq 0 .
$$

Take now any circuit $C$ in $D=(V, A)$ in the support of $\boldsymbol{x}^{d i r, k}$. Since we have supposed that $\boldsymbol{x}^{d i r, k}$ is not zero and since it is a circulation, such a circuit necessarily exists. According to Equations (9) and (10), we have $\mu_{a}^{d i r, k}=0$ for each $a \in C$. The sum $\sum_{a \in C} e_{a}^{k}$ is nonzero since no tree $T^{k}$ can contain all arcs in $C$. Summing Equation (8) for all arcs in $C$, we get

$$
\omega^{d i r}=-\frac{\sum_{a \in C} \alpha_{a}^{k} \sum_{k^{\prime} \in K} x_{a}^{d i r, k^{\prime}}}{\sum_{a \in C} e_{a}^{k}}<0 .
$$

It is in contradiction with Equation (10). It implies that $x_{a}^{d i r, k}=0$ for all $k \in K$ and $a \in A^{k}$.

We show now that $\pi_{v}^{d i r, k}=0$ for all $v \in V^{k}$. We start by noting that Equation (8) becomes

$$
\pi_{u}^{d i r, k}-\pi_{v}^{d i r, k}-\mu_{u v}^{d i r, k}=0, \quad \text { for all } k \in K \text { and }(u, v) \in T^{k} .
$$

Since $T^{k}$ is an $s^{k}$-arborescence, we have $0=\pi_{s^{k}}^{d i r, k} \geq \pi_{v}^{d i r, k}$ for all $v \in V^{k}$, according to Equation (10).

Define now $F^{k}$ to be the set of $\operatorname{arcs} a \in A^{k}$ such that $(a, k) \in B^{x}$. Using Remark 1 of Section 3.1, $\bar{M}_{B}^{e}$ has a nonzero entry on each of its first $\sum_{k \in K}\left(\left|V^{k}\right|-1\right)$ rows, which implies that the set $F^{k}$ spans all vertices in $V^{k} \backslash\left\{s^{k}\right\}$.

According to the non-degeneracy assumption, $\bar{x}_{a}^{k}$ is non-zero on all arcs of $F^{k}$. The complementarity condition for all points of the ray give that $\overline{\boldsymbol{x}} \cdot \boldsymbol{\mu}^{d i r}+\boldsymbol{x}^{d i r} \cdot \overline{\boldsymbol{\mu}}=0$, and since $\boldsymbol{x}^{d i r}=\mathbf{0}$, we have $\overline{\boldsymbol{x}} \cdot \boldsymbol{\mu}^{d i r}=0$. Hence $\mu_{u v}^{d i r, k}=0$ for all $(u, v) \in F^{k}$, and Equation (8) becomes

$$
\pi_{u}^{d i r, k}-\pi_{v}^{d i r, k}+e_{u v}^{k} \omega^{d i r}=0 \quad \text { for all } k \in K \text { and }(u, v) \in F^{k} .
$$

Thus, according to Equation (10), we have $0=\pi_{s^{k}}^{d i r, k} \leq \pi_{v}^{d i r, k}$ for all $v \in V^{k}$. Since we have already shown the reverse inequality, we have $\pi_{v}^{d i r, k}=0$ for all $v \in V^{k}$. 
Now, if $T^{k} \neq F^{k}$ for at least one $k$, we get the existence of an $\operatorname{arc}(u, v) \in F^{k}$ for which $e_{u v}^{k}=1$, while $\pi_{u}^{d i r, k}=\pi_{v}^{d i r, k}=0$. Equation (11) implies then that $\omega^{d i r}=0$. Still using $\boldsymbol{x}^{d i r}=\mathbf{0}$, we get then, again with the help of Equation (8), that $\boldsymbol{\mu}^{d i r}=\mathbf{0}$, which contradicts the fact that $\rho$ is an infinite ray.

Therefore, we have $T^{k}=F^{k}$ for all $k$. Using Remark 2 of Section 3.5, we are at the initial basic solution: $B=B^{i n i}$. According to Equation (8), and since $\boldsymbol{x}^{d i r}=\mathbf{0}$ and $\boldsymbol{\pi}^{d i r}=\mathbf{0}$, we have $\mu_{u v}^{d i r, k}=e_{u v}^{k} \omega^{d i r}$ for all $k \in K$ and $(u, v) \in A^{k}$. Thus $\left(\boldsymbol{x}^{d i r}, \boldsymbol{\mu}^{d i r}, \omega^{d i r}, \boldsymbol{\pi}^{d i r}\right)=\omega^{d i r}(\mathbf{0}, \boldsymbol{e}, 1, \mathbf{0})$ for $\omega^{d i r} \geq 0$, and $\rho$ is necessarily the primary ray $\rho^{i n i}$.

Then there is no secondary ray, as required.

Université Paris Est, CERMICS (ENPC), F-77455 MARne-LA-VAllÉE

E-mail address: frederic.meunier@enpc.fr, thomas.pradeau@enpc.fr 